\title{
Prognostic significance of bone marrow histological evaluation in patients with multiple myeloma
}

\author{
Andrzej Szczepaniak ${ }^{1 *}$, Maciej Kaźmierczak ${ }^{1}$, Mieczysław Komarnicki ${ }^{1}$, \\ Anna Przybyłowicz-Chalecka ${ }^{1}$, Violetta Filas ${ }^{2}$, Michał Michalak ${ }^{3}$, Lidia Gil ${ }^{1}$ \\ ${ }^{1}$ Department of Hematology and Bone Marrow Transplantation, Poznan University of Medical Sciences Poznan, Poland \\ ${ }^{2}$ Department of Tumor Pathology and Prophylaxis, Poznan University of Medical Sciences, Poznan, Poland \\ ${ }^{3}$ Department of Computer Science and Statistics, Poznan University of Medical Sciences, Poznan, Poland
}

\begin{abstract}
Introduction: Recently, there has been enormous progress in the diagnosis and treatment of multiple myeloma (MM). Despite the importance of flow cytometry and cytogenetics, biomarkers are being sought to assess prognosis and responses to treatment. The aim of this study was to determine the significance of histological evaluation and immunohistochemical markers in MM patients.
\end{abstract}

Material and methods: A retrospective analysis was performed in 90 bone marrow (BM) biopsies collected during the diagnosis period and related to the recognized prognostic factors and response to treatment. Standardized staining methods and monoclonal antibodies were used to detect clusters of differentiation antigens - CD138, CD56, CD20, CD117, cyclin D1, p53, kappa and lambda light chains.

Results: During the follow-up period of 49.6 (2-119.7) months in patients with increased levels of $\beta_{2}$-microglobulin, higher ISS (International Prognostic Classification) stage, with resistance to induction and higher relapse rate after first-line, a clonal lambda plasmocytes infiltration was observed. It also affected the response to induction with immunomodulatory drugs (IMiDs), but not bortezomib. The lowest response rate to IMiDs was noted in patients with CD117-lambda+ and CD56-lambda+ plasma cells. Clonal lambda hyperplasia was connected with a reduction in overall survival [hazard ratio (HR) 2.4; 95\% confidence interval (Cl): 0.96-5.92, $p=0.047$ ] and in progression-free survival (PFS) (HR 4.32, 95\% Cl: 1.9-9.5, $p=0.0002$ ). The presence of plasmocytes with phenotype CD117-lambda+ (HR 3.37; 95\% Cl: $1.57-7.24, p=0.002$ ) and CD56-lambda+ (HR 3.44; 95\% Cl: 1.63-7.27, $p=0.001$ ) contributed to PFS shortening. Conclusion: BM biopsy with immunohistochemistry allows the identification of poorly prognostic patients with MM. Key words: multiple myeloma, histology, immunohistochemistry, prognosis

Acta Haematologica Polonica 2021; 52, 5: 493-503

\section{Introduction}

Multiple myeloma (MM) is a disease of plasma cells neoplasms. It is the third commonest cancer of the lymphoid tissue in adults in Poland according to the number of new cases. According to the criteria updated in 2014, the diagnosis is based on the presence of clonal infiltration of pathological plasma cells in a tissue biopsy or bone marrow (BM) (at least $10 \%$ of cells) and the presence of at least one of the symptoms that defines myeloma [1, 2]. The methods for assessing the neoplastic character of plasma cells include immunohistochemistry (IHC), immunophenotyping,

\footnotetext{
*Address for correspondence: Andrzej Szczepaniak, Department of Hematology and Bone Marrow Transplantation, Poznan University of Medical Sciences, Szamarzewskiego 84, Poznan 60-569, Poland, phone +486185493 83, e-mail: ajjszczepaniak@gmail.com 
and cytogenetic tests. The evaluation of clonal character is based on the presence of the disturbed proportion of kappa positive to lambda positive plasmocytes based on the IHC study of trephine biopsies and the aberrant phenotype of plasma cells. Despite the increased importance of flow cytometry (FC) in recent years [3], histological examination of the bone marrow remains the basic method to assess the percentage of infiltration (which can be underestimated in the FC assessment), fibrosis and the pattern of neoplastic infiltration $[4,5]$. In addition, the use of the extended panel of IHC antibodies allows a more accurate analysis of bone marrow involvement and the distinguishing of prognostic factors at the diagnostic biopsy.

The aim of this study was to determine the diagnostic usefulness of selected parameters of histological evaluation of bone marrow and immunohistochemical markers in a group of patients with newly diagnosed (NDMM) or refractory/relapsed MM (RRMM), as well as the determination of histological prognostic factors in trephine biopsies of NDMM.

\section{Material and methods}

A group of 202 patients diagnosed with multiple myeloma treated at the Department of Hematology and Bone Marrow Transplantation at the Medical University of Poznan, Poland in 2008-2016 was included in an initial analysis. The inclusion criterion was a diagnosis of MM according to the 2014 International Myeloma Working Group (IMWG) definition in adult patients. The exclusion criteria from the study were the coexistence of another neoplastic disease, or a diagnosis of plasma cell (PC) leukemia and bone marrow infiltration by histopathology of below $10 \%$. Eventually, 90 patients with MM met all of the above criteria (47 women and 43 men), with median age 60 years (range 32-86).

Archival trephine biopsies obtained from the posterior superior iliac spine, collected during the standard diagnostics of newly diagnosed $(n=56)$ or refractory/relapsed ( $n=34$ ) MM patients, were prepared and stained by standard methods in the Cancer Pathology Department of the Greater Poland Cancer Center in Poznan (Table I). Microscopic evaluation was made by an experienced pathologist according to the recommendations of the International Council for Standardization in Hematology in 2008. The histological parameters of the bone marrow were analyzed for the percentage of plasmocytes based on hematoxylin and eosin $(\mathrm{H}+\mathrm{E})$ staining and immunohistochemical staining for the presence of CD138 antigen (cluster of differentiation), distribution pattern (three types of infiltration were distinguished: diffuse/clusters of plasmocytes/interstitial) and cytomorphological type of infiltration (plasmablastic and immature) [6]. For cytomorphological verification, each time the bone marrow smear from BM aspirate was assessed, thus confirming the diagnosis of MM. The grade of marrow fibrosis (MF) was described on a four-point scale (MF0-MF3) based on histochemical staining for the presence of reticulin fibers [7]. The following IHC stains were evaluated: CD138, CD56, CD20, CD117, cyclin D1, p53 protein, kappa and lambda light chains to determine the clonality and to reveal the aberrant phenotype of myeloma cells (Table I) [8-15]. In this study, we use the term kappa or lambda plasma cells infiltration if the kappa positive to lambda positive cells ratio in IHC was $>4: 1$ or $<1: 2$ respectively. In addition, the analysis included clinical data (date of diagnosis, initiation of therapy, disease progression, last observation/death, type of first line therapy, and response to induction therapy), laboratory [monoclonal protein class, immunoglobulin light chain type, albumin, beta ${ }_{2}-$ microglob- $^{-}$ ulin $(\beta 2 \mathrm{M})$ and creatinine concentration, lactate dehydrogenase activity] and cytogenetic aberrations [minimum panel by IMWG included t(4;14), t(14;16), del(17p) [16] (Table II).

The median observation time was 49.6 (2-119.7) months.

Firstly, a comparative analysis of patients with newly diagnosed or refractory/relapsed disease was performed in terms of clinical, laboratory and histological parameters. Afterwards, to determine the prognostic value, a statistical analysis of the association between histological parameters and well defined prognostic factors (Table III) [17-20] was performed using the Pearson chi-square independence test and Fisher's exact test. The assessment of overall survival (OS) and progression-free survival (PFS) were made using Kaplan-Meier curves, which were then compared using the Log-rank test. To determine the factors affecting the OS and PFS, the Cox proportional hazard regression model was used with the determination of the hazard ratio (HR) with a 95\% confidence interval $(\mathrm{Cl})$. All tests were evaluated at the significance level, which was $p<0.05$.

The study protocol was approved by the Local Bioethics Committee at the University of Medical Sciences in Poznan, and was carried out with accordance with Declaration of Helsinki.

\section{Results}

The bone marrow cellularity was higher in the group of newly diagnosed patients than in those with refractory/relapsed disease. However, there were no statistically significant differences in the percentage of infiltration, the distribution pattern, the histological type of infiltration, the fibrosis stage or the frequency of immunohistochemical markers expression ( $p>0.05$ ) (Table IV).

Analyzing the group of 56 patients with newly diagnosed MM, an association between the type of neoplastic infiltration and the severity of fibrosis, as well as the Durie-Salmon stage, was observed. In patients with diffuse neoplastic infiltration, bone marrow fibrosis $(p=0.001)$ and 
Table I. Methodology of histological and immunohistochemical staining

Average length of analyzed biopsies

$14.1 \mathrm{~mm}$ (range 9-28)

Trephine biopsy preparation

Tissue material was fixed in 10\% buffered formalin at $\mathrm{pH} 7.4$; then placed in 10\% formic acid for $24 \mathrm{~h}$; transferred to a tissue processor; prepared tissue embedded in paraffin at $60^{\circ} \mathrm{C}$ using standard histopathological methods; from the obtained paraffin blocks, sections 4-5 $\mu \mathrm{m}$ thick were cut on a microtome, placed on adhesive slides, and left for $1 \mathrm{~h}$ at $60^{\circ} \mathrm{C}$

Histological staining for morphological assessment

Hematoxylin and eosin staining

Staining for presence of reticulin fibrosis

BENCHMARK Special Stains Stainer Module by Ventana Medical System/Roche Diagnostics

Immunohistochemical markers determination (clone of antibody; kit and IHC method; IP staining)

\begin{tabular}{l|l} 
Cyclin D1 & Rabbit anti-cyclin D1 from Roche (clone SP4-R); IHC method with use of ultraView Universal DAB
\end{tabular} Detection Kit by Roche; IP on BenchMark UItra from VentanaMedical System/Roche Diagnostics

$\mathrm{CD56}$

CD138

Monoclonal RTU and polyclonal antibodies from DAKO: murine antibodies anti-CD56 (IR642), anti-

CD117 -CD138 (IR642) and rabbit polyclonal antibodies concentrated against: CD117 kit (A4502), lambda light chains (A0193) and kappa light chains (A0191); IHC method with use of DAKO En VisionTM

Lambda LC FLEX+ system, antigens were detected in paraffin sections in DAKO's Target Retrieval Solution, High pH buffer in a PT-link apparatus at $97^{\circ} \mathrm{C}, 20$ min.; IP staining DAKO Autostainer Link 48 apparatus

Kappa LC

CD20

Murine monoclonal antibodies from DAKO: anti-CD20 (GA604) and anti-p53 (GA616); IHC method p53 with use of DAKO En VisionTM FLEX + system; IP staining was performed in OMNIS apparatus by DAKO

Cut-off percentage for positive result $(+)$

\begin{tabular}{|c|c|c|}
\hline & Literature data* & Mean values in research group \\
\hline CD56 & 50 & 40 \\
\hline CD20 & 10 & 20 \\
\hline CD117 & 5 & 10 \\
\hline p53 & 10 & $>0$ \\
\hline cyclin D1 & 10 & 30 \\
\hline CD138 & 10 & 10 \\
\hline LC kappa/lambda & \multicolumn{2}{|c|}{ kappa $[+] /$ lambda[+] cells ratio $>4: 1$ or $<1: 2$} \\
\hline
\end{tabular}

*[8-15]; IHC - immunohistochemical; IP - immunoperoxidase; LC - light chain

a more advanced stage of disease were more common $(p=0.006)$. Among patients with unfavorable cytogenetic aberrations, the immature and plasmablastic type of tumor infiltration was observed more frequently (with del17p vs. without del $17 p-21.4 \%$ vs. $2.3 \%$ respectively; $p=0.044$; with $\mathrm{t}(4 ; 14)$ vs. without $\mathrm{t}(4 ; 14)-28.6 \%$ vs. $4.1 \%$ respectively; $p=0.049)$. With an increasing percentage of bone marrow involvement ( $<20 \%$ vs. $20-50 \%$ vs. $>50 \%$ ), the occurrence of diffuse pattern versus clusters and interstitial type of infiltration ( $p=0.001$ ) and higher stage of fibrosis $(p=0.006)$ were observed more often. The analysis of significance of IHC markers revealed that the presence of high expression of CD56 ( $\geq 50 \%$ of cells) correlated with the occurrence of $\mathrm{t}(4 ; 14)(p=0.046)$. Moreover, CD20 expression ( $\geq 20 \%$ of cells) was connected with a diffuse pattern of tumor infiltration ( $p=0.026$ ) and a higher stage of bone marrow fibrosis ( $p=0.004$ ). The presence of the lambda immunoglobulin light chain on the clonal plasmocytes was associated with an elevated $\beta 2 \mathrm{M}$ concentration ( $p=0.017)$, a higher ISS stage ( $p=0.04)$, a lack of response to induction therapy ( $p=0.009)$, and more frequent relapses after the first line of treatment ( $p=0.006$ ) (Table V).

A histological risk factor for OS shortening was the presence of lambda plasma cells infiltration (HR 2.4, 95\% Cl: 0.96-5.92, $p=0.047$ ) (Figure 1). Lambda positive patients achieved median survival (42.3 months) in contrast to kappa positive ones, whereas the finding of plasmocytes with the CD117-kappa+ and CD56-kappa+ phenotype was associated with longer survival (HR 0.25, 95\% Cl: 0.09$-0.72, p=0.01$ and HR 0.24, 95\% Cl: 0.08-0.75, $p=0.014$, 
Table II. Characteristics of study group at time of diagnosis

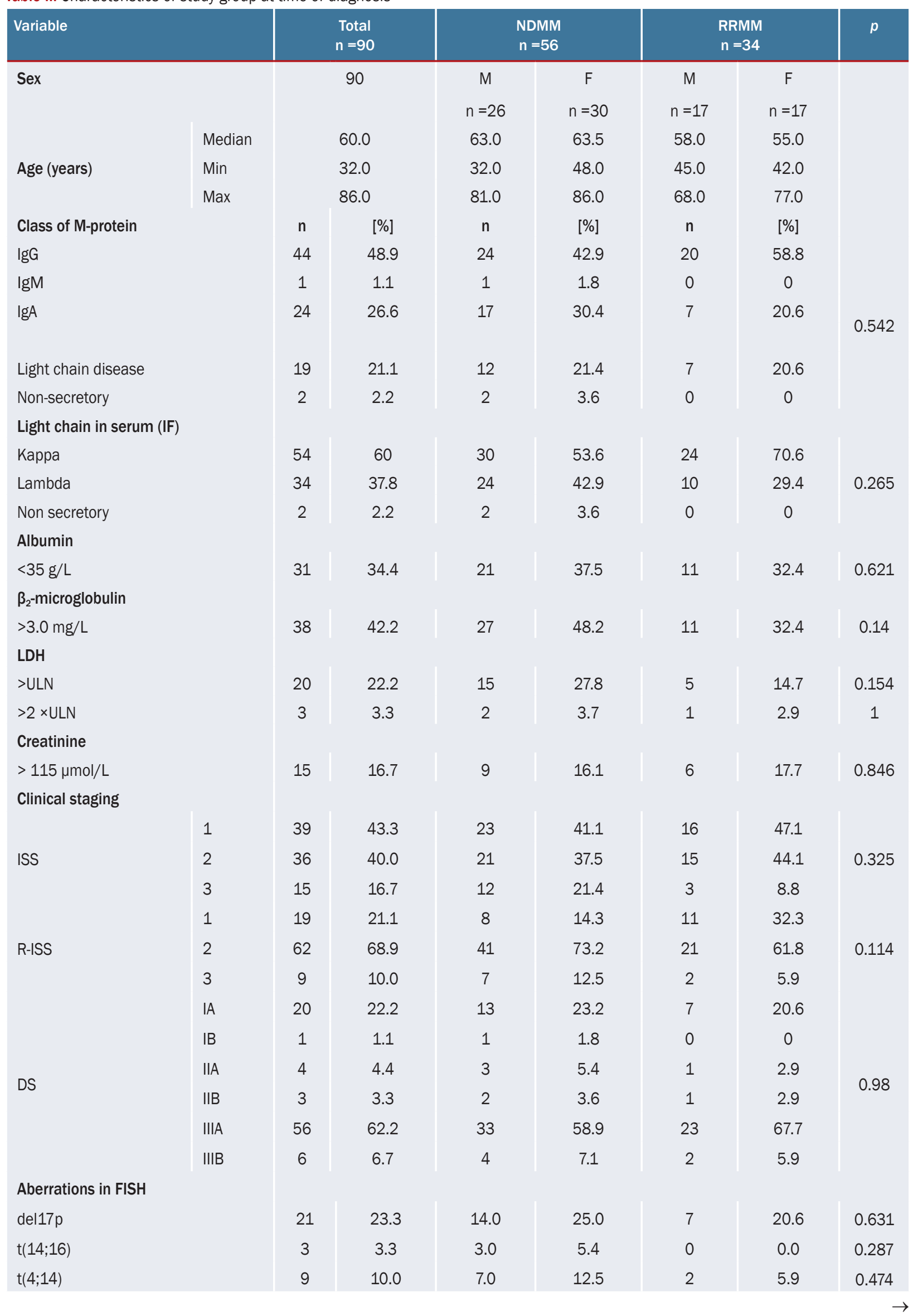


Table II (cont.). Characteristics of study group at time of diagnosis

\begin{tabular}{|c|c|c|c|c|c|c|c|}
\hline Variable & \multicolumn{2}{|c|}{$\begin{array}{l}\text { Total } \\
\mathrm{n}=90\end{array}$} & \multicolumn{2}{|c|}{$\begin{array}{c}\text { NDMM } \\
\mathrm{n}=56\end{array}$} & \multicolumn{2}{|c|}{$\begin{array}{l}\text { RRMM } \\
n=34\end{array}$} & $p$ \\
\hline \multicolumn{8}{|c|}{ Classification by Rajkumar } \\
\hline Standard & 58 & 64.4 & 36.0 & 64.3 & 22 & 64.7 & \multirow{3}{*}{0.451} \\
\hline Medium & 9 & 10.0 & 4.0 & 7.1 & 5 & 14.7 & \\
\hline High & 23 & 25.6 & 16.0 & 28.6 & 7 & 20.6 & \\
\hline \multicolumn{8}{|c|}{ Classification mSMART } \\
\hline Standard & 54 & 60.0 & 30 & 53.6 & 24 & 70.6 & \multirow{3}{*}{0.318} \\
\hline Medium & 12 & 13.3 & 9.0 & 16.1 & 3 & 8.8 & \\
\hline High & 24 & 26.7 & 17.0 & 30.4 & 7 & 20.6 & \\
\hline
\end{tabular}

NDMM - newly diagnosed multiple myeloma; RRMM - refractory/relapsed multiple myeloma; M - male; F - female; IgG - immunoglobulin G; IgM - immunoglobulin M; IgA - immunoglobulin A; LDH - lactate dehydrogenase; ULN - upper limit of normal; ISS - International Staging System; R-ISS - Revised International Staging System; DS - Durie-Salmon Staging System; FISH - fluorescence in situ hybridization; mSMART - Mayo Stratification of Myeloma and Risk-Adapted Therapy

Table III. Defined prognostic factors in multiple myeloma, in reference to which importance of histological parameters was determined

\begin{tabular}{|c|c|}
\hline \multicolumn{2}{|l|}{ Prognostic factor } \\
\hline $\begin{array}{l}\text { Cytogenetic aber- } \\
\text { rations in FISH }\end{array}$ & del17p, t(14;16), t(4;14) \\
\hline $\begin{array}{l}\text { Cytogenetic risk } \\
\text { groups }\end{array}$ & mSMART [17] and by Rajkumar [18] \\
\hline Histological para- & Fibrosis grade \\
\hline meters & Distribution pattern \\
\hline & Histological type of infiltration \\
\hline Laboratory para- & Type of monoclonal protein \\
\hline meters & $\begin{array}{l}\text { Type of serum light chain in electrop- } \\
\text { horesis }\end{array}$ \\
\hline & $\downarrow$ albumin concentration \\
\hline & $\uparrow \beta 2 \mathrm{M}$ concentration \\
\hline & $\uparrow$ creatinine concentration \\
\hline & $\uparrow$ LDH activity \\
\hline Stage of disease & $\begin{array}{l}\text { by Durie-Salmon, ISS, R-ISS classifica- } \\
\text { tion }[19,20]\end{array}$ \\
\hline Clinical response & Relapse after first-line treatment \\
\hline & Lack of response to first-line treatment \\
\hline
\end{tabular}

FISH - fluorescence in situ hybridization; mSMART - Mayo Stratification of Myeloma and Risk-Adapted Therapy; $\beta 2 \mathrm{M}$ - beta ${ }_{2}$-macroglobulin; LDH - lactate dehydrogenase; ISS - International Staging System; R-ISS - Revised International Staging System

respectively). The presence of clonal lambda infiltration (HR $4.32,95 \% \mathrm{Cl}: 1.9-9.5, p=0.0002$ ), as well as the presence of plasmocytes with the CD117-lambda+ (HR 3.37, 95\% Cl: 1.57-7.24, $p=0.002$ ) and CD56-lambda+ phenotype (HR 3.44, 95\% Cl 1.63-7.27, $p=0.001$ ), were determined as immunohistochemical risk factors affecting PFS in the study group (Figure 2).
In the group of patients with newly diagnosed MM, the overall response rate (ORR) to the first line treatment was $69.1 \%$ (38/56 patients), and was a factor favorably affecting OS (HR 0.39; 95\% Cl: 0.16-0,95, $p=0.038$ ). In addition, it was demonstrated that the presence of tumor cells expressing the lambda chain was a factor negatively affecting the response to induction therapy with immunomodulatory drugs (IMiDs) (ORR $36.4 \%$ vs. $86.9 \%$ for kappa patients; $p=0.005$ ). The lowest percentage of responses to IMiDs was found in patients with CD117-lambda+ $(p=0.009)$ and CD56-lambda $+(p=0.014)$ tumor cells phenotype. The above correlations were not observed in the group treated with bortezomib. It has been shown, however, that patients with increased CD20 expression achieved a response to bortezomib treatment $(p=0.022)$ statistically less frequently.

\section{Discussion}

Despite the development of modern techniques based on immunophenotypic and molecular tests, BM biopsy remains an integral part of diagnostics and often determines the diagnosis of MM. The main advantage of histological bone marrow assessment is the unique ability to determine parameters such as cellularity, bone marrow architecture, grade of fibrosis or angiogenesis. Moreover, it influences the possibility of numerous specific immunohistochemical staining and stability of the bone biopsies.

The disadvantages of histological evaluation include a certain subjective evaluation, depending on the experience of the pathologist and the amount of material available for analysis. The assessment of the percentage of bone marrow infiltration (>10\%) according to the IMWG criteria [2] is crucial for the diagnosis of MM, and the fact of underestimation in the BM smear or cytometry has been confirmed [21]. Pathological plasmocytes are disproportionately hemodiluted compared to other bone marrow cells, 
Table IV. Comparison of frequency of assessed histological parameters and immunohistochemical markers

\begin{tabular}{|c|c|c|c|c|c|}
\hline \multirow{2}{*}{ Histological parameter } & \multicolumn{2}{|c|}{ NDMM $(n=56)$} & \multicolumn{2}{|c|}{ RRMM ( $n=34)$} & \multirow[t]{2}{*}{$p$} \\
\hline & $\mathrm{n}$ & [\%] & $\mathrm{n}$ & [\%] & \\
\hline \multicolumn{6}{|l|}{ Cellularity of bone marrow } \\
\hline Hypercellular & 48 & 85.7 & 21 & 61.8 & \multirow{3}{*}{0.04} \\
\hline Mediumcellular & 6 & 10.7 & 11 & 32.1 & \\
\hline Hypocellular & 2 & 3.6 & 2 & 5.9 & \\
\hline \multicolumn{6}{|l|}{ Fibrosis } \\
\hline MFO & 33 & 58.9 & 21 & 61.8 & \multirow{4}{*}{0.547} \\
\hline MF1 & 18 & 32.1 & 8 & 23.5 & \\
\hline MF2 & 5 & 8.9 & 5 & 14.7 & \\
\hline MF3 & 0 & 0.0 & 0 & 0.0 & \\
\hline \multicolumn{6}{|l|}{ Distribution pattern } \\
\hline Diffuse & 17 & 30.4 & 7 & 20.6 & \multirow{3}{*}{0.281} \\
\hline Clusters of plasmocytes & 26 & 46.4 & 14 & 41.9 & \\
\hline Interstitial & 13 & 23.2 & 13 & 38.2 & \\
\hline \multicolumn{6}{|c|}{ Histological type of infiltration } \\
\hline Immature, plasmablastic & 4 & 7.1 & 3 & 8.8 & 1 \\
\hline \multicolumn{6}{|l|}{ Degree of infiltration } \\
\hline$<20 \%$ & 6 & 10.7 & 9 & 26.5 & \multirow{3}{*}{0.094} \\
\hline $20-50 \%$ & 18 & 32.1 & 12 & 35.3 & \\
\hline$>50 \%$ & 32 & 57.1 & 13 & 38.2 & \\
\hline \multicolumn{6}{|c|}{ Expression of IHC markers (\% cut off for positive result) } \\
\hline $\operatorname{CD56+}(\geq 40 \%)$ & 16 & 28.6 & 10 & 29.4 & 0.932 \\
\hline CD56+ ( $\geq 50 \%)$ & 14 & 25.0 & 9 & 26.5 & 0.877 \\
\hline$C D 20+(\geq 10 \%)$ & 15 & 26.8 & 7 & 20.6 & 0.507 \\
\hline$C D 20+(\geq 20 \%)$ & 9 & 16.1 & 4 & 11.8 & 0.573 \\
\hline $\mathrm{p} 53+(>0)$ & 5 & 8.9 & 4 & 11.8 & 0.725 \\
\hline$p 53+(\geq 10 \%)$ & 2 & 3.6 & 2 & 5.9 & 0.631 \\
\hline CD117+ ( $\geq 5 \%)$ & 16 & 28.6 & 8 & 23.5 & 0.6 \\
\hline $\mathrm{CD} 117+(\geq 10 \%)$ & 12 & 21.4 & 7 & 20.6 & 0.925 \\
\hline Cyclin D1 ( $\geq 10 \%)$ & 37 & 66.1 & 24 & 70.6 & 0.657 \\
\hline Cyclin D1 ( $\geq 30 \%)$ & 26 & 46.4 & 15 & 44.1 & 0.831 \\
\hline Kappa positive & 32 & 57.1 & 24 & 70.6 & 0.202 \\
\hline Lambda positive & 24 & 42.9 & 10 & 29.4 & 0.202 \\
\hline
\end{tabular}

NDMM - newly diagnosed multiple myeloma; RRMM - refractory/relapsed multiple myeloma

and have other physical and biological properties that influence cytometric results. In addition, the myeloma infiltration may appear as clusters, as in other lymphoproliferations. A biopsy with $\mathrm{CD} 138$ assessment may be decisive in those cases, as confirmed by Al-Quaran et al. [8] and Lee et al. [22]. The percentage of BM infiltration by clonal PC is not only a diagnostic, but also a poor prognostic, factor influencing OS and PFS [23].
The negative importance of the plasmablastic type of infiltration is known to be an independent prognostic factor for survival at the time of diagnosis and additionally affects OS and PFS in primary refractory patients and those relapsed after autotransplant [24]. Patients with plasmablastic hyperplasia significantly more often have clonal cytogenetic abnormalities (73\% vs. $31 \%$ ). Our study confirmed a more frequent occurrence of clonal, poor cytogenetic aberrations 
Table V. Correlation between clonal light chain expression on plasma cells and clinical parameters in group of patients with newly diagnosed myeloma

\begin{tabular}{|c|c|c|c|c|c|}
\hline \multirow{2}{*}{$\begin{array}{l}\text { Light chain expression and selec- } \\
\text { ted parameters }\end{array}$} & \multicolumn{2}{|c|}{ Kappa } & \multicolumn{2}{|c|}{ Lambda } & \multirow[t]{2}{*}{$p$} \\
\hline & $\mathrm{n}$ & [\%] & $\mathrm{n}$ & [\%] & \\
\hline \multicolumn{6}{|l|}{ Beta $_{2}$-macroglobulin } \\
\hline$>3.0 \mathrm{mg} / \mathrm{L}$ & 11 & 34.4 & 16 & 66.7 & 0.017 \\
\hline$<3.0 \mathrm{mg} / \mathrm{L}$ & 21 & 65.6 & 8 & 33.3 & \\
\hline \multicolumn{6}{|l|}{ Response to first-line treatment } \\
\hline Yes & 27 & 84.4 & 12 & 52.2 & 0.009 \\
\hline No & 5 & 15.6 & 11 & 47.8 & \\
\hline \multicolumn{6}{|l|}{ Relapse after first-line treatment } \\
\hline Yes & 9 & 28.1 & 14 & 66.7 & 0.006 \\
\hline No & 23 & 71.9 & 7 & 33.3 & \\
\hline \multicolumn{6}{|l|}{ International Staging System } \\
\hline ISS 1 & 17 & 53.1 & 6 & 25 & 0.04 \\
\hline ISS 2 & 8 & 25 & 13 & 54.2 & \\
\hline ISS 3 & 7 & 21.9 & 5 & 20.8 & \\
\hline
\end{tabular}
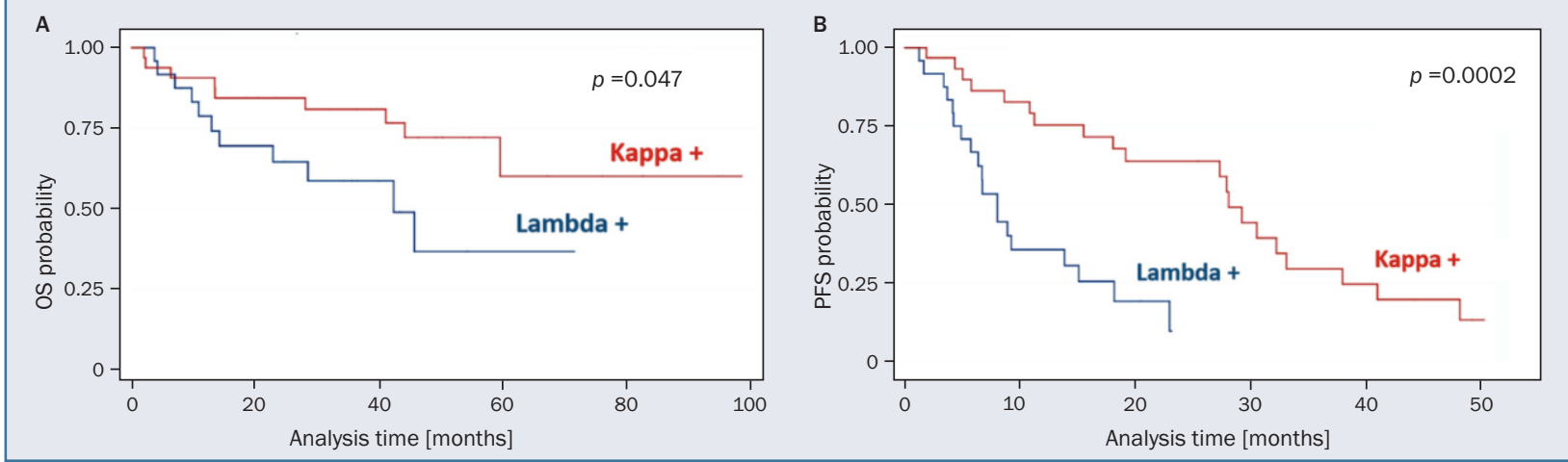

Figure 1. Kaplan-Meier analysis of overall survival (OS) (A) and progression-free survival (PFS) (B) in relation to immunoglobulin light chain expression on plasma cells

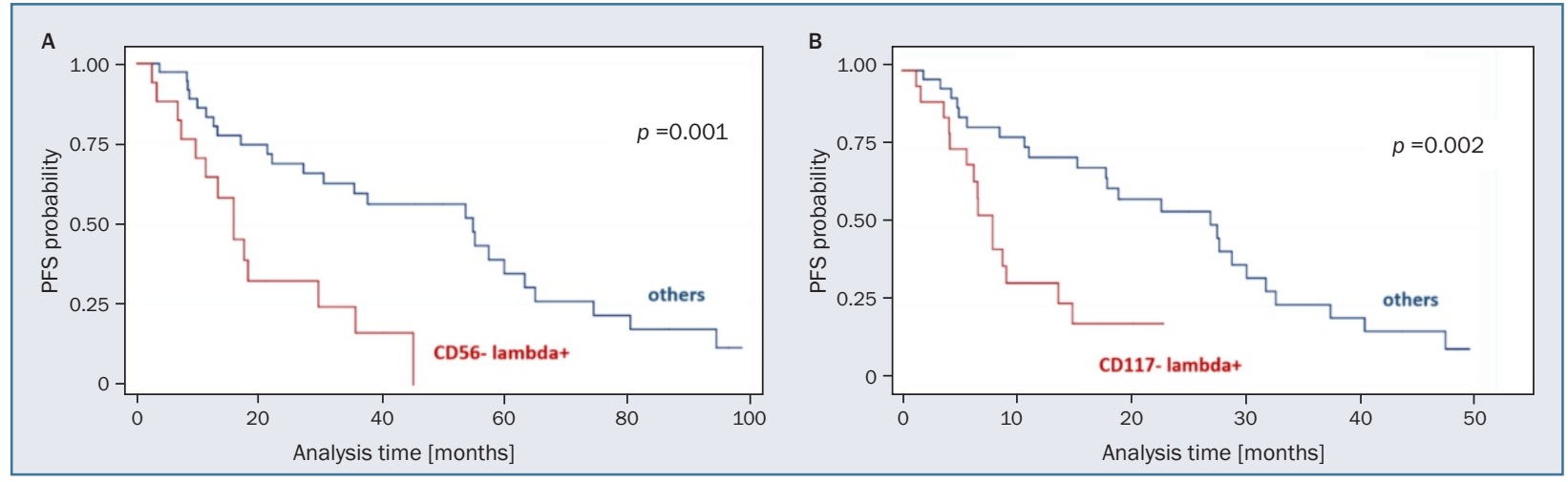

Figure 2. Kaplan-Meier analysis of progression-free survival (PFS) in relation to plasma cells phenotype: CD56-lambda+ $(p=0.0006)(A)$ and CD117-lambda+ $(p=0.002)(B)$ 
(del17p) and $t(4 ; 14)$ in the group with plasmablastic infiltration, as well as shorter OS in contrast to other morphological types (5 vs. 18 months). However, due to the small size of the group, statistical significance was not achieved.

Histology of bone marrow is the only technique to evaluate fibrosis. This parameter is widely discussed in the literature in the context of many hematological diseases, although most of the published data concerns myeloproliferative neoplasms. Bone marrow fibrosis is also important in relation to patients with $\mathrm{MM}$. Our data corresponds to that of the available studies, where fibrosis was found in $20-38 \%$ of MM cases. It has been described that myeloma with advanced bone marrow fibrosis and only immunoglobulin light chains has a more aggressive course. Additionally, the appearance of excess fibrosis correlates with a higher degree and more diffuse type of infiltration by plasmocytes. Hallgrimsdottir et al. proved in nearly 600 cases that patients with fibrosis had a significantly worse OS compared to a group without fibrosis (OS 4.4 vs. 5 years, respectively) $[6,25,26]$. Survival analysis in relation to fibrosis in our study did not show statistically significant differences in the group with NDMM. However, the median OS of 59.4 months was achieved only among patients with severe fibrosis (MF2) and PFS was significantly shorter (8.9 months) compared to patients without (MF0, 19.1 months) or with mild (MF1) fibrosis (18 months).

To date, many studies have been carried out on IHC markers on myeloma cells, but only a few published studies present such a broad panel of antigens in the BM biopsies in MM patients as in our study.

The significance of CD56 is unclear. Patients with a lack of CD56 expression in FC more often had extramedullary localization, elevated Bence-Jones protein, renal failure, a more aggressive course, and worse OS, compared to the CD56 positive group [27], in contrast to similar analysis performed with immunohistochemical methods [28]. Pan et al. reported that a simultaneous lack of CD56 and CD117 expression was associated with a shorter OS [29]. We did not confirm that observation, but high CD56 expression was correlated with more frequent presence of an unfavorable aberration $(4 ; 14)$ and the presence of plasmocytes with the CD56-kappa+ phenotype positively influenced OS and PFS.

The presence of the c-KIT antigen (CD117) has been described in $20-35 \%$ of MM cases and increased expression is related to a better prognosis [30]. Bataille et al. [31] described the expression of CD117 in MGUS (monoclonal gammopathy of undetermined significance) patients to be significantly higher compared to MM ones and found that the loss of CD117 on cells was associated with disease progression. It has been suggested that expression of CD117 on monoclonal plasmocytes alters their target site in the BM and may direct them to neutrophil precursor niches where CD117 acts as an anchor molecule. Such redistribution of monoclonal plasma cells in the bone marrow may contribute to a greater maintenance of the homeostatic role of residual normal plasmocytes and their more limited spread in CD117+ monoclonal gammopathies [32]. Other analyzes of NDMM patients showed a statistically worse prognosis, easier expansion of plasma cells, and a shorter OS in the group without expression of both CD56 and CD117 [29, 33].

The importance of CD20 in patients with myeloma is not fully understood, and the literature data shows that this group is heterogeneous. Robillard et al. described that expression of CD20 in MM patients was associated with $t(11 ; 14)$, the morphology of small, mature plasmocytes and a more favorable prognosis [34]. In contrast, Quinn et al. [35] distinguished a population with concomitant expression of CD20 and cyclin D2 which was characterized by a more aggressive course. The CD20+ myeloma cells were the most common in the form of diffuse infiltration [35]. This last observation was also confirmed in our study. Increased presence of CD20 without CD56 expression has also been observed in plasma cell leukemia, with a particularly unfavorable prognosis [36].

Monoclonal light chains (LC) immunoglobulin, detected by immunohistochemical staining, allow the differentiation of diseases from the other plasma cell dyscrasias, and are present in the cytoplasm of myeloma cells in approximately $85 \%$ of cases. The immunoglobulin light chain determined by IHC correlated with the serum LC, clinical diagnosis and was confirmed as an independent negative prognostic factor for the progression of smoldering $\mathrm{MM}$, as well as for NDMM [37, 38]. Patients with light chain lambda show a significantly shorter survival (median OS 10 vs. 30 months for lambda and kappa respectively) as described by Shustik et al. [39]. Expression of the lambda chain was associated with a predisposition to extramedullary localization and rapidly progressive renal failure $[40,41]$.

Our study confirms a correlation of lambda infiltration with increased $\beta 2 \mathrm{M}$ concentration, higher ISS, and poor response to first line treatment in NDMM. The analysis showed that the clonal lambda cells negatively affected OS and PFS. In addition, the presence of plasma cells with phenotype CD117-lambda+ and CD56-lambda+ was specified as a new, poor, prognostic factor affecting PFS. The type of clonal PC infiltration also determines the response to treatment. The introduction of IMiDs into MM therapy improved OS, and kappa infiltration was one of the factors positively influencing the ORR $[42,43]$. The use of IMiDs, however, does not overcome the negative impact of the unfavorable cytogenetic or clonal lambda hyperplasia. On the contrary, the use of a proteasome inhibitor, bortezomib, improved the response to treatment in the group of patients with $t(4 ; 14)$ or del17p $[44,45]$. The use of bortezomib in triplets [VTD (bortezomib, thalidomide, dexamethasone)] showed superiority in a group of patients with myeloma $\leq 65$ years 
eligible for autotransplantation, but also in a group of elderly people who were not eligible for transplant [46, 47]. This regimen statistically more often induced a complete response and was also active at relapse [48].

In our study, no differences were found in the response rate to bortezomib treatment depending on the unfavorable cytogenetic profile and the type of light chain. Therefore the use of bortezomib seems to be especially indicated in high-risk patients.

\section{Conclusions}

Based on the performed analyzes, the diagnostic and prognostic usefulness of histological assessment with immunohistochemistry in patients with MM was confirmed. At the diagnostic biopsy, the prognostic factors related to the course of the disease can be specified. The most important histological parameter adversely affecting overall survival and progression-free survival is the presence of lambda positive plasmocytes infiltration. Moreover, bortezomib has been shown to be particularly useful in the first line treatment in patients with lambda clonal plasma cells.

\section{Acknowledgements}

This project was co-financed by a grant from the Young Hematologist Club.

\section{Authors' contributions}

AS - study design, data collection and analysis, manuscript writing. $\mathrm{MKa}$ - conceived idea for manuscript, data check. MKo, AP-C, VF - laboratory work-up. MM - statistical analysis. All authors - final approval of manuscript.

\section{Conflict of interest}

None.

\section{Financial support}

None.

\section{Ethics}

The work described in this article has been carried out in accordance with The Code of Ethics of the World Medical Association (Declaration of Helsinki) for experiments involving humans; EU Directive 2010/63/EU for animal experiments; Uniform Requirements for manuscripts submitted to biomedical journals.

\section{References}

1. Dmoszyńska A, Usnarska-Zubkiewicz L, Walewski J, et al. Zalecenia Polskiej Grupy Szpiczakowej dotyczące rozpoznawania i leczenia szpiczaka plazmocytowego oraz innych dyskrazji plazmocytowych na rok 2017. Acta Haematol Pol. 2017; 48(2): 55-103, doi: 10.1016/j. achaem.2017.05.003.
2. Rajkumar S, Dimopoulos M, Palumbo A, et al. International Myeloma Working Group updated criteria for the diagnosis of multiple myeloma. Lancet Oncol. 2014; 15(12): e538-e548, doi: 10.1016/s14702045(14)70442-5.

3. Rawstron AC, Orfao A, Beksac M, et al. European Myeloma Network. Report of the European Myeloma Network on multiparametric flow cytometry in multiple myeloma and related disorders. Haematologica. 2008; 93(3): 431-438, doi: 10.3324/haematol.11080, indexed in Pubmed: 18268286.

4. Swerdlow SH, Harris NL, Jaffe ES, Pileri SA, Stein H, Thiele J. WHO Classification of tumours of haematopoietic and lymphoid tissues. 4th ed. Vol. 2. IARC Publication, Lyon 2017.

5. Matsue K, Matsue Y, Kumata K, et al. Quantification of bone marrow plasma cell infiltration in multiple myeloma: usefulness of bone marrow aspirate clot with CD138 immunohistochemistry. Hematol Oncol. 2017; 35(3): 323-328, doi: 10.1002/hon.2300, indexed in Pubmed: 27140172.

6. Jaffe ES, Arber DA, Campo E, Harris NL, Quintanilla-Martinez L. Hematoathology. Elsevier, Philadelphia 2016: 474-493.

7. Thiele J, Kvasnicka F, Facchetti F, et al. European consensus on grading bone marrow fibrosis and assessment of cellularity. Haematologica, 2005. Haematologica. 2005; 90(8): 1128-1132.

8. Al-Quran SZ, Yang L, Magill JM, et al. Assessment of bone marrow plasma cell infiltrates in multiple myeloma: the added value of CD138 immunohistochemistry. Hum Pathol. 2007; 38(12): 1779-1787, doi: 10.1016/j.humpath.2007.04.010, indexed in Pubmed: 17714757.

9. Yeung J, Chang H. Genomic aberrations and immunohistochemical markers as prognostic indicators in multiple myeloma. J Clin Pathol. 2008; 61(7): 832-836, doi: 10.1136/jcp.2007.049585, indexed in Pubmed: 18077770.

10. Ng AP, Wei A, Bhurani D, et al. CD 138 immunostaining of bone marrow trephine specimens is the most sensitive method for quantifying marrow involvement in patients with plasma cell dyscrasias. Blood. 2005; 106(11): 5071-5071, doi: 10.1182/blood.v106.11.5071.5071.

11. Chang H, Samiee S, Yi QiL. Prognostic relevance of CD56 expression in multiple myeloma: a study including 107 cases treated with high-dose melphalan-based chemotherapy and autologous stem cell transplant. Leuk Lymphoma. 2006; 47(1): 43-47, doi: 10.1080/10428190500272549, indexed in Pubmed: 16321826.

12. Yavasoglu I, Sargin G, Kadikoylu G, et al. Immunohistochemical evaluation of CD20 expression in patients with multiple myeloma. Rev Bras Hematol Hemoter. 2015; 37(1): 34-37, doi: 10.1016/j. bjhh.2014.11.013, indexed in Pubmed: 25638765.

13. Goto K, Takai T, Fukumoto T, et al. CD117 (KIT) is a useful immunohistochemical marker for differentiating porocarcinoma from squamous cell carcinoma. J Cutan Pathol. 2016; 43(3): 219-226, doi: 10.1111/ cup.12632, indexed in Pubmed: 26449497.

14. Padhi S, Varghese RG, Ramdas A. Cyclin D1 expression in multiple myeloma by immunohistochemistry: case series of 14 patients and literature review. Indian J Med Paediatr Oncol. 2013; 34(4): 283-291, doi: 10.4103/0971-5851.125246, indexed in Pubmed: 24604959.

15. Chng WJ, Price-Troska T, Gonzalez-Paz N, et al. Clinical significance of TP53 mutation in myeloma. Leukemia. 2007; 21(3): 582-584, doi: 10.1038/sj.leu.2404524, indexed in Pubmed: 17215851.

16. Fonseca R, Bergsagel PL, Drach J, et al. International Myeloma Working Group. International Myeloma Working Group molecular classification of multiple myeloma: spotlight review. Leukemia. 2009; 23(12): 2210-2221, doi: 10.1038/leu.2009.174, indexed in Pubmed: 19798094. 
17. Mikhael JR, Dingli D, Roy V, et al. Mayo Clinic. Management of newly diagnosed symptomatic multiple myeloma: updated Mayo Stratification of Myeloma and Risk-Adapted Therapy (mSMART) consensus guidelines 2013. Mayo Clin Proc. 2013; 88(4): 360-376, doi: 10.1016/j. mayocp.2013.01.019, indexed in Pubmed: 23541011.

18. Rajan AM, Rajkumar SV. Interpretation of cytogenetic results in multiple myeloma for clinical practice. Blood Cancer J. 2015; 5: e365, doi: 10.1038/bcj.2015.92, indexed in Pubmed: 26517360.

19. Greipp PR, San Miguel J, Durie BGM, et al. International staging system for multiple myeloma. J Clin Oncol. 2005; 23(15): 3412-3420, doi: 10.1200/JC0.2005.04.242, indexed in Pubmed: 15809451.

20. Palumbo A, Avet-Loiseau H, Oliva S, et al. Revised International Staging System for Multiple Myeloma: a Report from International Myeloma Working Group. J Clin Oncol. 2015; 33(26): 2863-2869, doi: 10.1200/JC0.2015.61.2267, indexed in Pubmed: 26240224.

21. Tran DN, Smith SA, Brown DA, et al. Polychromatic flow cytometry is more sensitive than microscopy in detecting small monoclonal plasma cell populations. Cytometry B Clin Cytom. 2017; 92(2): 136-144, doi: 10.1002/cyto.b.21401, indexed in Pubmed: 27416483.

22. Lee N, Moon SY, Lee JH, et al. Discrepancies between the percentage of plasma cells in bone marrow aspiration and BM biopsy: Impact on the revised IMWG diagnostic criteria of multiple myeloma. Blood Cancer J. 2017; 7(2): e530, doi: 10.1038/bcj.2017.14, indexed in Pubmed: 28211888.

23. Rajkumar SV, Fonseca R, Dispenzieri A, et al. Methods for estimation of bone marrow plasma cell involvement in myeloma: predictive value for response and survival in patients undergoing autologous stem cell transplantation. Am J Hematol. 2001; 68(4): 269-275, doi: 10.1002/ ajh.10003, indexed in Pubmed: 11754416.

24. Rajkumar SV, Fonseca R, Lacy MQ, et al. Plasmablastic morphology is an independent predictor of poor survival after autologous stem-cell transplantation for multiple myeloma. J Clin Oncol. 1999; 17(5): 1551-1557, doi: 10.1200/JC0.1999.17.5.1551, indexed in Pubmed: 10334543.

25. Hallgrimsdottir T, Porwit A, Björkholm M, et al. Bone marrow fibrosis in patients with multiple myeloma: a new prognostic factor for survival? Blood. 2013; 122(21): 1946-1946, doi: 10.1182/blood. v122.21.1946.1946.

26. Subramanian R, Basu D, Dutta TK. Significance of bone marrow fibrosis in multiple myeloma. Pathology. 2007; 39(5): 512-515, doi: 10.1080/00313020701570038, indexed in Pubmed: 17886102.

27. Sahara N, Takeshita A, Shigeno K, et al. Clinicopathological and prognostic characteristics of CD56-negative multiple myeloma. Br J Haematol. 2002; 117(4): 882-885, doi: 10.1046/j.13652141.2002.03513.x, indexed in Pubmed: 12060125.

28. Yeung J, Samiee S, Yi Q, et al. Prognostic relevance of CD56 expression in patients with multiple myeloma treated with high-dose melphalan-based chemotherapy and autologous stem cell transplant. Blood. 2005; 106(11): 5086-5086, doi: 10.1182/blood.v106.11.5086.5086.

29. Pan $\mathrm{Y}$, Wang $\mathrm{H}$, Tao Q, et al. Absence of both CD56 and CD117 expression on malignant plasma cells is related with a poor prognosis in patients with newly diagnosed multiple myeloma. Leuk Res. 2016; 40: 77-82, doi: 10.1016/j.leukres.2015.11.003, indexed in Pubmed: 26597998.

30. Mateo G, Castellanos M, Rasillo A, et al. Genetic abnormalities and patterns of antigenic expression in multiple myeloma. Clin Cancer Res. 2005; 11(10): 3661-3667, doi: 10.1158/1078-0432.CCR-04-1489, indexed in Pubmed: 15897562.
31. Bataille R, Pellat-Deceunynck C, Robillard N, et al. CD117 (c-kit) is aberrantly expressed in a subset of MGUS and multiple myeloma with unexpectedly good prognosis. Leuk Res. 2008; 32(3): 379-382, doi: 10.1016/j.leukres.2007.07.016, indexed in Pubmed: 17767956 .

32. Schmidt-Hieber M, Pérez-Andrés M, Paiva B, et al. CD117 expression in gammopathies is associated with an altered maturation of the myeloid and lymphoid hematopoietic cell compartments and favorable disease features. Haematologica. 2011; 96(2): 328-332, doi: 10.3324/haematol.2010.031872, indexed in Pubmed: 20971816.

33. Ceran F, Falay M, Dağdaş S, et al. The assessment of CD56 and CD117 expressions at the time of the diagnosis in multiple myeloma patients. Turk J Haematol. 2017; 34(3): 226-232, doi: 10.4274/ tjh.2016.0394, indexed in Pubmed: 28270374.

34. Robillard N, Avet-Loiseau H, Garand R, et al. CD2O is associated with a small mature plasma cell morphology and $\mathrm{t}(11 ; 14)$ in multiple myeloma. Blood. 2003; 102(3): 1070-1071, doi: 10.1182/ blood-2002-11-3333, indexed in Pubmed: 12702507.

35. Quinn J, Percy L, Glassford J, et al. CD20-positive multiple myeloma - differential expression of cyclins D1 and D2 suggests a heterogeneous disease. Br J Haematol. 2010; 149(1): 156-159, doi: 10.1111/j.1365-2141.2009.08030.x, indexed in Pubmed: 19995388.

36. Fernández de Larrea C, Kyle RA, Durie BGM, et al. International Myeloma Working Group. Plasma cell leukemia: consensus statement on diagnostic requirements, response criteria and treatment recommendations by the International Myeloma Working Group. Leukemia. 2013; 27(4): 780-791, doi: 10.1038/leu.2012.336, indexed in Pubmed: 23288300.

37. Dispenzieri A, Kyle RA, Katzmann JA, et al. Immunoglobulin free light chain ratio is an independent risk factor for progression of smoldering (asymptomatic) multiple myeloma. Blood. 2008; 111(2): 785-789, doi: 10.1182/blood-2007-08-108357, indexed in Pubmed: 17942755

38. Kyrtsonis MC, Vassilakopoulos TP, Kafasi N, et al. Prognostic value of serum free light chain ratio at diagnosis in multiple myeloma. Br J Haematol. 2007; 137(3): 240-243, doi: 10.1111/j.13652141.2007.06561.x, indexed in Pubmed: 17408464.

39. Shustik C, Bergsagel DE, Pruzanski W. Kappa and lambda light chain disease: survival rates and clinical manifestations. Blood. 1976; 48(1): 41-51, doi: 10.1182/blood.v48.1.41.41.

40. Kozlowski P, Montgomery S, Befekadu R, et al. The risk of renal disease is increased in lambda myeloma with bone marrow amyloid deposits. J Blood Med. 2017; 8: 29-34, doi: 10.2147/JBM.S129516, indexed in Pubmed: 28293126.

41. Shameem M, Akhtar J, Bhargava R, et al. Lambda light chain multiple myeloma presenting as pleural mass. Respiratory Medicine CME. 2011; 4(1): 12-14, doi: 10.1016/j.rmedc.2010.01.007.

42. Morgan GJ, Davies F, Gregory W, et al. The addition of thalidomide to the induction treatment of newly presenting myeloma patients increases the $\mathrm{CR}$ rate which is likely to translate into improved PFS and OS. Blood. 2009; 114(22): 352-352, doi: 10.1182/blood. v114.22.352.352.

43. Munehiko A, Sameshima Y, Oda T, et al. [Factors affecting the response of thalidomide therapy for patients with multiple myeloma] [Article in Japanese]. Rinsho Ketsueki. 2010; 51(3): 189-195, indexed in Pubmed: 20379113. 
44. Avet-Loiseau H, Leleu X, Roussel M, et al. Bortezomib plus dexamethasone induction improves outcome of patients with $t(4 ; 14)$ myeloma but not outcome of patients with del(17p). J Clin Oncol. 2010; 28(30): 4630-4634, doi: 10.1200/JC0.2010.28.3945, indexed in Pubmed: 20644101.

45. El-Ghammaz AMS, Abdelwahed E. Bortezomib-based induction improves progression-free survival of myeloma patients harboring $17 \mathrm{p} \mathrm{de}$ letion and/or $\mathrm{t}(4 ; 14)$ and overcomes their adverse prognosis. Ann Hematol. 2016; 95(8): 1315-1321, doi: 10.1007/s00277-016-2692-0, indexed in Pubmed: 27184486.

46. San Miguel JF, Schlag R, Khuageva NK, et al. VISTA Trial Investigators. Bortezomib plus melphalan and prednisone for initial treatment of multiple myeloma. N Engl J Med. 2008; 359(9):
906-917, doi: 10.1056/NEJMoa0801479, indexed in Pubmed: 18753647.

47. Rosiñol L, Oriol A, Teruel Al, et al. Programa para el Estudio y la Terapéutica de las Hemopatías Malignas/Grupo Español de Mieloma (PETHEMA/GEM) group. Superiority of bortezomib, thalidomide, and dexamethasone (VTD) as induction pretransplantation therapy in multiple myeloma: a randomized phase 3 PETHEMA/GEM study. Blood. 2012; 120(8): 1589-1596, doi: 10.1182/blood-2012-02-408922, indexed in Pubmed: 22791289.

48. Tacchetti P, Pantani L, Stefano VDe, et al. Superior PFS2 with VTD vs TD for newly diagnosed, transplant eligible, multiple myeloma (MM) patients: updated analysis of Gimema MMY-3006 study. Blood. 2014; 124(21): 196-196, doi: 10.1182/blood.v124.21.196.196. 